\title{
Contribution of different pneumococcal virulence factors to experimental meningitis in mice
}

\author{
Susanna Ricci ${ }^{1 *}$, Alice Gerlini ${ }^{1}$, Andrea Pammolli ${ }^{2}$, Damiana Chiavolini ${ }^{1,5}$, Velia Braione ${ }^{1,6}$, Sergio Antonio Tripodi ${ }^{3}$, \\ Bruna Colombari ${ }^{4}$, Elisabetta Blasi ${ }^{4}$, Marco Rinaldo Oggioni ${ }^{1}$, Samuele Peppoloni ${ }^{4}$ and Gianni Pozzi ${ }^{1}$
}

\begin{abstract}
Background: Pneumococcal meningitis (PM) is a life-threatening disease with a high case-fatality rate and elevated risk for serious neurological sequelae. In this study, we investigated the contribution of three major virulence factors of Streptococcus pneumoniae, the capsule, pneumococcal surface protein A (PspA) and C (PspC), to the pathogenesis of experimental PM.
\end{abstract}

Methods: Mice were challenged by the intracranial route with the serotype 4 TIGR4 strain (wt) and three isogenic mutants devoid of PspA, PspC, and the capsule. Survival, bacterial counts, and brain histology were carried out. To study the interaction between S. pneumoniae mutants and microglia, phagocytosis and survival experiments were performed using the BV2 mouse microglial cell line.

Results: Virulence of the PspC mutant was comparable to that of TIGR4. In contrast, survival of animals challenged with the PspA mutant was significantly increased compared with the wt, and the mutant was also impaired at replicating in the brain and blood of infected mice. Brain histology indicated that all strains, except for the unencapsulated mutant, caused PM. Analysis of inflammation and damage in the brain of mice infected with TIGR4 or its unencapsulated mutant demonstrated that the rough strain was unable to induce inflammation and neuronal injury, even at high challenge doses. Results with BV2 cells showed no differences in phagocytic uptake between wt and mutants. In survival assays, however, the PspA mutant showed significantly reduced survival in microglia compared with the wt.

Conclusions: PspA contributed to PM pathogenesis possibly by interacting with microglia at early infection stages, while PspC had limited importance in the disease. The rough mutant did not cause brain inflammation, neuronal damage or mouse death, strengthening the key role of the capsule in PM.

Keywords: Experimental pneumococcal meningitis, Microglia, PspA, PspC, Capsule

\section{Background}

Streptococcus pneumoniae is a coloniser of the human nasopharynx and can also cause other diseases, including sinusitis, otitis media, pneumonia, sepsis and meningitis. The microorganism produces a plethora of virulence factors, including the polysaccharide capsule, several surface-located proteins, and the toxin pneumolysin [1,2]. The capsule is a major virulence determinant due to its anti-phagocytic activity [3-5]. Among the surface-associated proteins, the pneumococcal surface protein A (PspA) and

\footnotetext{
* Correspondence: susanna.ricci@unisi.it

'Department of Medical Biotechnologies, Laboratory of Molecular Microbiology and Biotechnology (LA.M.M.B.), University of Siena and Siena University Hospital, Siena 53100, Italy

Full list of author information is available at the end of the article
}

$\mathrm{C}(\mathrm{PspC})$ are the best characterised choline-binding proteins. PspA interferes with complement activation and deposition mediated by both the classical and alternative pathways [6-9] and also binds lactoferrin [10]. PspC interacts with human immunoglobin $\mathrm{A}$ and with the polymeric immunoglobulin receptor $[11,12]$, thereby promoting adhesion and transcytosis of pneumococci across mucosal surfaces $[13,14]$. PspC also shows anti-phagocytic properties due to its capability to bind to complement C3 [15] and factor $\mathrm{H}[11,16-18]$.

Pneumococcal meningitis (PM) is a life-threatening disease with high rates of mortality and neurological sequelae $[19,20]$. The hallmark of meningitis is represented by cerebrospinal fluid (CSF) pleocytosis, which largely

\section{Biomed Central}


contributes to brain inflammation and damage [20,21]. It is well accepted that meningitis-induced brain injury depends on both the host inflammatory response and the direct bacterial toxicity [19-21]. The pneumococcal cell wall and pneumolysin initiate immune activation in the CSF by engaging the toll-like receptors 2 and 4, respectively [22]. Peptidoglycan and teichoic acid have been shown to trigger meningeal inflammation in experimental PM [23,24]. Pneumolysin interferes with the beat frequency of brain ependymal cilia [25], damages the blood-brain-barrier (BBB) [26], mediates apoptosis of microglial and neuronal cells in vitro [27] and participates in hearing loss and cochlear damage associated with experimental PM [28]; moreover, a pneumolysindeficient mutant showed reduced virulence in murine PM [29]. Other pneumococcal knock-out mutants have been analysed in PM models, including strains devoid of the neuroaminidases NanA and NanB [29], the hyaluronidase [29] and the fibronectin-binding protein PavA [30]. To our knowledge, so far the role of PspA and PspC has not been assessed in experimental PM.

Microglial cells, comprising about $15 \%$ of brain cells, are located within the brain parenchyma and constitute the main phagocytic population of the central nervous system (CNS) [31,32]. Activated microglia can wield several effector functions, such as phagocytosis, inflammatory responses and antigen presentation [32]. During infection of the CNS, microglial cells secrete proinflammatory mediators involved in the recruitment of peripheral immune cells to the site of infection and also exert antimicrobial activity towards invading pathogens [33]. Microglia is therefore considered a key player in the initial innate immune response against CNS infections.

In the present study, we tested the serotype 4 TIGR4 strain and three isogenic mutants deficient in PspA, PspC and capsule in an intracranic mouse model of PM. We also focused on anti-phagocytic pneumococcal virulence determinants and on microglial cells, key effectors of innate immunity and first line of defence against $S$. pneumoniae invading the brain, especially in the early phases of PM.

\section{Methods}

\section{Bacterial strains and growth conditions}

S. pneumoniae TIGR4 (type 4) and the isogenic mutants FP23 (rough), FP28 (PspC-) and FP262 (PspA-) were used in this work. Bacteria were grown in Tryptic Soy Broth (TSB, Becton Dickinson, Milano, Italy) until midlogarithmic phase and stored at $-80^{\circ} \mathrm{C}$ with $10 \%$ glycerol. Solid media were prepared by addition of $1.5 \%$ agar and 3\% defibrinated horse blood (Oxoid, Hampshire, UK) to TSB. Counts of colony forming units (cfu) were performed on blood-agar plates at $37^{\circ} \mathrm{C}$ with $5 \%$
$\mathrm{CO}_{2}$. When necessary, chloramphenicol, erythromycin and kanamycin were used at the concentrations of $2.5 \mu \mathrm{g} / \mathrm{ml}, 1 \mu \mathrm{g} / \mathrm{ml}$ and $500 \mu \mathrm{g} / \mathrm{ml}$, respectively.

\section{Construction of knock-out mutants}

All mutant strains were generated by gene SOEing [34]. Construction of the unencapsulated derivative of TIGR4 (FP23) and of the PspC-deficient mutant (FP28) has already been described [35,36]. To construct the PspAdeficient strain, the $p s p A$ gene was replaced with an erythromycin-resistance cassette $(\mathrm{ermB})$ [37] using primers IF188 (5' -AAGTGATTTGTGATTGTTGATG$3^{\prime}$ ) and IF189 (5'-ACCTCTTTAGCTCCTTGGAAG-3') [38]. Primer pairs employed to amplify the regions upstream (845 bp) and downstream (587 bp) of the pspA gene were IF215 (5' ${ }^{\prime}$ TTGGGCAGTAGTGAGAACTG$\left.3^{\prime}\right) /$ IF216 (5' -CATCAACAATCACAAATCACTTCAG ACTATACTTATATTAAG-3') and IF217 (5' -CTTCCA AGGAGCTAAAGAGGTGCCGATTAAATTAAAGCAT G-3')/ IF218 (5'-ATCTTCGGTCGCCGTACAGA-3'), respectively. A $2571 \mathrm{bp}-$ long PCR fragment was used to transform TIGR4, and an erythromycin-resistance mutant was selected and designated as strain FP262. Mutant construction was verified by PCR and sequencing.

\section{Mice, model of meningitis and experimental design}

Outbred 8 to 10-week-old female MF1 mice (Harlan Nossan, Monza, Italy) were used. Animal experimentation was approved by the local ethical committee, and all experiments were performed according to institutional and national guidelines ('Ministero della Salute', Decreto no. 72/2012-B). The method to induce PM in mice has been previously reported [39]. Briefly, mice were lightly anesthetised by intraperitoneal (i.p.) injection of xylazine hydrochloride (Bio 98 S.r.l., Bologna, Italy) and zolazepam tiletamine (Virbac S.r.l., Milano, Italy) and inoculated by the intracranial route (i.c.) with $50 \mu \mathrm{l}$ of the bacterial inoculum using a micro-syringe with 26 gauge needles (Hamilton, Bonaduz, Switzerland). Studies on survival and cfu determinations in tissues were conducted on animal groups infected with $10^{2}, 10^{3}$ and $10^{4} \mathrm{cfu} /$ mouse. For the rough strain FP23, rodents were also infected with larger doses up to $10^{7} \mathrm{cfu} /$ mouse. Animal group sizes are provided in Additional file 1: Tables S1, S2 and S3. Mice were monitored twice a day for clinical signs as described by Sandgren et al. [40]. Briefly, disease severity was graded using endpoints on a scale of $0-5$, with $0=$ normal, $1=$ piloerection and decreased spontaneous activity, $2=$ hunched position and loss of vigilance, $3=$ turns upright in $>5 \mathrm{sec}$ when positioned on the back, $4=$ does not turn upright, $5=$ moribund. Mice were euthanised if/ when they reached a score of 4 . Body weight and temperature were recorded once per day for 10 days and 
compared to those of naïve uninfected control mice. Survival was recorded for 10 days. Assessment of PM by histology was carried out on the brain of animals $(n=3$ / group) infected with $10^{4} \mathrm{cfu} /$ mouse of $\mathrm{S}$. pneumoniae strains and sacrificed $48 \mathrm{~h}$ post-infection. For histological evaluation of the role of capsule in PM, two groups of mice were infected with $10^{5} \mathrm{cfu} /$ mouse of TIGR4 $(n=6)$ or FP23 $(n=16)$ and sacrificed after 24 and $48 \mathrm{~h}$ (3-8 mice/time point). To strengthen the results obtained with the dose of $10^{5} \mathrm{cfu}$, another group of mice $(n=4)$ was challenged with $10^{7}$ cfu of FP23 and sacrificed $72 \mathrm{~h}$ post-infection. Control mice were inoculated with $50 \mu \mathrm{l}$ of phosphate buffered saline (PBS).

\section{Sample collection}

Blood and brain were collected from infected mice for histological analysis and cfu counts. Blood samples were obtained by the sub-mandibular vein. For cfu counts, $100 \mathrm{U} / \mathrm{ml}$ of heparin (MS Pharma, Milano, Italy) were added to blood samples to prevent coagulation, whereas brains were homogenised in $1 \mathrm{ml}$ of TSB. Blood and brain samples were frozen at $-80^{\circ} \mathrm{C}$ with $10 \%$ glycerol until use. Bacterial counts were performed by plating 10-fold dilutions onto blood-agar plates.

\section{Brain histology}

For histological analysis, brains were immediately fixed in formalin for $24 \mathrm{~h}$ and then embedded in paraffin according to standard procedures. The brains were entirely sectioned along a coronal plane. Sections were stained with haematoxylin-eosin according to standard techniques. The presence and degree of inflammation and neuronal damage were evaluated by using routine light microscopy (at least 100 power fields were examined). Inflammation was estimated by counting the number of polymorphonuclear cells (PMN) in four different brain regions: superficial meningeal regions over the convexities, frontal interhemispheric region, hippocampal fissure and third ventricle. Based on the number of PMN for each power field, a score was attributed as follows: 0 (0 PMN), 1 (<10 PMN), 2 (10-50 PMN) and 3 ( $>50$ PMN). For every animal, scores of each brain region were summed up into a final inflammation score (IS). Neuronal damage was evaluated by estimating the percent of damaged neurons in one power field and calculating a score as follows: 0 (no damaged neurons), 1 $(<10 \%), 2(10-30 \%)$ and $3(>30 \%)$. Both apoptotic and necrotic neurons were considered injured. Apoptosis was represented by cell shrinkage, homogenous chromatin condensation, nuclear shrinkage and nuclear transformation into apoptotic bodies. Cell swelling, eosinophilic degeneration of the cytoplasm, nuclear shrinkage with chromatin clumping were considered signs of necrosis. Four different brain areas were analysed: neocortex, striatum, hippocampus/dentate gyrus and cerebellum. Scores of each brain region for each mouse were summed, and the resulting number represented the final damage score (DS).

\section{Microglial cells}

The murine microglial cell line BV2 [41] was maintained in RPMI 1640 medium supplemented with 10\% heat-inactivated fetal calf serum (hiFCS) (Defined Hyclone, Logan, UT, USA), gentamicin $(50 \mu \mathrm{g} / \mathrm{ml}$; Bio Whittaker, Verviers, Belgium) and L-glutamine (2 mM; EuroClone, Milan, Italy) (complete medium). Cells were detached biweekly by vigorous shaking, and fresh cultures were started at a concentration of $5 \times 10^{5} / \mathrm{ml}$.

\section{Phagocytosis assay}

For all fluorescence-based assays, pneumococci were thawed, washed and suspended at the desired concentrations. Staining of bacteria was performed by incubating $10^{8} \mathrm{cfu} / \mathrm{ml}$ with $5 \mathrm{mM}$ of Hoechst 33342 (SigmaAldrich, St. Louis, MO, USA) in the dark at $37^{\circ} \mathrm{C}$ for $1 \mathrm{~h}$ as described $[42,43]$. After labelling, pneumococci were washed four times with PBS and then suspended at the desired concentration in complete RPMI medium without antibiotics. To strengthen the attachment of BV2 cells to wells, Lab-Tek II chamber slides (Nalge Nunc International, Naperville, IL, USA) were pretreated with poly-L-lysine (Sigma-Aldrich; $10 \mu \mathrm{g} /$ well) for $30 \mathrm{~min}$ and then washed with PBS. BV2 cells $\left(10^{6} / \mathrm{ml}, 100 \mu \mathrm{l} /\right.$ well $)$ were seeded, incubated for $15 \mathrm{~min}$ and infected (moi = 10) with $100 \mu \mathrm{l}$ of $10^{7} / \mathrm{ml}$ cfu of Hoechst 33342-labelled $S$. pneumoniae in RPMI containing L-glutamine and normal FCS (nFCS). After incubation for $3 \mathrm{~h}$, cells were treated with trypan blue for 5 min to quench the fluorescence of bound bacteria, washed with PBS to remove extracellular bacteria and fixed for 30 min with $4 \%$ paraformaldehyde (PFA) (Sigma-Aldrich) in PBS. Finally, BV2 cells were washed with PBS and treated with ProLong Gold Antifade Reagent (Molecular Probes, Invitrogen, St. Louis, Mo, USA) to suppress the photobleaching effect and preserve the signals of fluorescent molecules. Remaining fluorescence of phagocytosed bacteria was visualised by epifluorescence microscopy. At least 200 microglial cells from each sample were examined, and the percentage of cells with intracellular bacteria was defined as the ratio of the number of BV2 cells containing one or more bacteria to the total number of cells examined.

\section{Phagolysosome acidification assay}

Visualisation of bacteria-containing acidic phagosomes was performed as described [42,43]. Briefly, BV2 cells were infected for $3 \mathrm{~h}$ (see phagocytosis assay), washed to eliminate extracellular bacteria, and exposed to $4 \mu \mathrm{l}$ 
of the acidotropic dye LysoTracker Red DND-99 (Molecular Probes, Invitrogen) at a final concentration of $5 \mu \mathrm{M}$. Thirty minutes before the end of incubation, an additional volume $(4 \mu \mathrm{l})$ of the same dye was added. Finally, $5 \mathrm{~min}$ before the end of incubation, $100 \mu \mathrm{l}$ of trypan blue were dispensed into each well. After PFA fixing, BV2 cells were washed and treated with ProLong Gold Antifade Reagent (Molecular Probes, Invitrogen). Acidification of phagosomes containing Hoechst 33342-labelled bacteria was visualised by epifluorescence microscopy by the simultaneous appearance of LysoTracker Red DND-99 (red) and Hoechst 33342 (blue) fluorescence within the phagosomes, resulting in purple fluorescence when merging images. For quantitative analysis, the number of bacteria-containing acidic phagosomes per image was determined by counting the number of purple phagosomes within phagocytic cells. The percentage of colocalisation was then calculated as the number of cells with bacteria-containing acidic phagosomes over the total number of phagocytic cells.

\section{Epifluorescence microscopy}

Prior to visualisation, Lab-Tek II chamber slides were washed with PBS and treated with Prolong Gold antifade Reagent (Molecular Probes, Invitrogen). Epifluorescence and differential interference contrast (DIC) microscopy were performed using a Nikon Eclipse 90i imaging system equipped with Nomarski DIC optics (Nikon Instruments Inc., Melville, NY, USA). Samples were photographed with a DS-2Mv Nikon digital camera, and the resulting photographs were analysed by using the Nikon NIS-ELEMENTS version D3.1 software.

\section{Intracellular survival assay}

Bacterial survival inside microglial cells was assessed by performing an antibiotic-protection assay as previously described $[42,43]$. Briefly, BV2 cells $\left(10^{6} / \mathrm{ml}\right)$ were incubated for $3 \mathrm{~h}$ with bacteria $(\mathrm{moi}=10)$ in RPMI with nFCS. Cells were washed with PBS to remove extracellular bacteria and exposed for $1.5 \mathrm{~h}$ to gentamicin $(150 \mu \mathrm{g} / \mathrm{ml})$ and vancomycin $(10 \mu \mathrm{g} / \mathrm{ml})$ in RPMI with L-glutamine. BV2 cells were washed twice with PBS and suspended in complete RPMI without antibiotics (time 0 ). Following $4 \mathrm{~h}$ of incubation (time 4 ), cells were lysed with $0.2 \%(\mathrm{v} / \mathrm{v})$ Triton X-100 for $15 \mathrm{sec}$ to release intracellular bacteria, and serial dilutions of the lysates were plated onto blood-agar plates. After 36-48 h cfu were counted, and the survival index (SI) of each strain was calculated as the number of cfu at time $4 \mathrm{~h}$ divided by the number of cfu at time $0 \mathrm{~h}$. In all assays where microglial BV2 cells were exposed to $S$. pneumoniae, the viability of infected cells was tested and found comparable to that of uninfected control cells.

\section{Statistical analyses}

Detailed data on mouse survival are described in Additional file 1: Table S1 which reports the median survival time (h) with 95\% confidence interval (CI) for each mouse group together with the related statistical analysis (Log Rank test). Additional file 1: Tables S2 and S3 describe the mean and standard deviation (SD) of log cfu counts in the brain and blood of infected mice, respectively. Complete data on phagocytosis and intracellular survival of S. pneumoniae in BV2 microglial cells are reported (mean \pm SD) in Additional file 1: Table S4. Differences between TIGR4 and the mutants in Additional file 1: Tables S2, S3 and S4 were analysed by the Bootstrap ( $\mathrm{BCa}$ method) performed on 1000 stratified resampling [44]. Data on IS and DS from mice challenged with TIGR4 and FP23 are shown as median with interquartile range (IQR). Analysis of differences in DS and IS was carried out by the Mann-Whitney U test (Table 1 ). $P$ values $<0.05$ were considered as statistically significant.

\section{Results}

Analysis of virulence of TIGR4 and the isogenic mutants FP28 (PspC-), FP262 (PspA-) and FP23 (rough) was performed both in vivo in a PM mouse model and in vitro using a murine microglial cell line. Although a few results on FP23 have already been published [42], we still decided to include this strain in all experiments to provide the reader with a complete comparative view

Table 1 Brain inflammation and damage over time in mice infected with TIGR4 or FP23

\begin{tabular}{|c|c|c|c|c|c|c|}
\hline \multirow[t]{3}{*}{ Strain $^{a}$} & \multirow{2}{*}{\multicolumn{2}{|c|}{$\frac{\text { Inflammation score }}{\text { [median (IQR) }^{\mathbf{b}}}$}} & \multirow[t]{3}{*}{$p^{\mathrm{d}}$} & \multirow{2}{*}{\multicolumn{2}{|c|}{$\begin{array}{c}\text { Damage score } \\
\text { [median (IQR)] }^{c}\end{array}$}} & \multirow[t]{3}{*}{$p^{\mathrm{d}}$} \\
\hline & & & & & & \\
\hline & $24 \mathrm{~h}$ & $48 \mathrm{~h}$ & & $24 \mathrm{~h}$ & $48 \mathrm{~h}$ & \\
\hline \multirow[t]{2}{*}{ TIGR4 } & $2(2-6)$ & $12(2-12)$ & & $2(2-3)$ & $6(1-8)$ & \\
\hline & & & $<0.001$ & & & $<0.05$ \\
\hline FP23 & $0(0-0.75)$ & 0 & & $0(0-3)$ & $0(0-1.75)$ & \\
\hline
\end{tabular}

${ }^{a}$ Two groups of MF1 mice were infected via the i.c. route with $10^{5} \mathrm{cfu} / \mathrm{mouse}$ of TIGR4 $(n=6)$ or FP23 $(n=16)$. Animals were sacrificed at 24 or $48 h$, and brains were removed and treated for haematoxylin-eosin staining. Three (TIGR4) or 8 (FP23) mice per time-point were used.

Inflammation in the brain was evaluated by counting the number of PMN in one power field $(\times 40)$ and calculating a score as follows: 0 (0 PMN), $1(<10$ PMN), 2 (10-50 PMN) 3 (>50 PMN). Four different brain regions were analysed: superficial meningeal regions over the convexities, frontal interhemispheric region, hippocampal fissure and third ventricle. For every animal, scores of each brain region were summed up into a total inflammation score (IS). Results are expressed as the median IS with the interquartile range (IQR) for each mouse group at 24 and $48 \mathrm{~h}$.

${ }^{c}$ Neuronal damage was evaluated by estimating the percent of damaged neurons in one power field $(\times 40)$ and calculating a score as follows: 0 (no damaged neurons), 1 (<10\%), 2 (10-30\%), 3 (>30\%). Four different brain areas were analysed: neocortex, striatum, hippocampus/dentate gyrus and cerebellum. For each mouse, scores of each brain region were summed, and the resulting number represented the total damage score (DS). Results are expressed as the median DS with the interquartile range (IQR) for each mouse group at 24 and $48 \mathrm{~h}$.

${ }^{\mathrm{d}}$ Mann-Whitney $U$ test. Differences in inflammation and damage between the groups infected with TIGR4 and FP23 were analysed by combining IS and DS scores from all mice of each group, regardless of the euthanasia time. 
of the different behaviours of pneumococcal mutants devoid of three major virulence determinants.

\section{Analysis of mouse survival after infection with pneumococcal strains}

Based on previous data on lethal doses of S. pneumoniae killing $50 \%$ of animals $\left(\mathrm{LD}_{50}\right)$ in experimental PM [39], mice were infected by the i.c. route with different doses of S. pneumoniae strains TIGR4, FP28, FP262 and FP23. Analysis of mouse clinical parameters (body temperature and weight) showed no significant differences among the groups infected with the encapsulated strains (data not shown). All animals died following challenge with $10^{2} \mathrm{cfu}$ of TIGR4, while survival percentages of mice infected with FP28 and FP262 were 21 and 36, respectively (Figure 1A). At $10^{3} \mathrm{cfu} / \mathrm{mouse}$, survival dropped to 0 and $23 \%$ in the groups infected with FP28 and FP262, respectively (Figure 1A). No animals survived challenge with $10^{4} \mathrm{cfu}$ of TIGR4, FP28 and FP262 (Figure 1A). The $\mathrm{LD}_{50}$ of all strains, except for FP23, were below $10^{2} \mathrm{cfu} /$ mouse. Upon time-to-death analysis, mice infected with the PspA-deficient strain FP262 showed significantly prolonged survival at all doses (Figure $1 \mathrm{~B}$ and Additional file 1: Table S1, $p<0.01)$. In order to unravel differences among the groups, a Kaplan-Meyer analysis was carried out only on data from mice infected with the smallest dose of $10^{2} \mathrm{cfu}$. Median survival times of rodents challenged with TIGR4, FP28, FP262 and FP23 were 48, 56, 144 and $>240$ h with significant differences for the groups TIGR4-FP262 and TIGR4-FP23 (Figure $1 \mathrm{~B}$ and Additional file 1: Table S1, $p<0.01$ for both groups). In accordance with previously published data [42], mice injected with the rough mutant FP23 presented clinical signs comparable to uninfected naïve animals and survived all challenge doses.

\section{Replication of pneumococcal strains in the brain and blood of mice following i.c. infection}

Analysis of the growth features of the mutants in standard media showed no differences compared with TIGR4 (data not shown).To evaluate their capability to replicate in the CNS, viable counts were determined over time (at 6,24 and $48 \mathrm{~h}$ ) in the brain of infected mice. No differences in bacterial titers were observed between FP28 and TIGR4 at any time-point. In contrast, the FP262 bacterial load in the brain was significantly lower than that of TIGR4 at $24 \mathrm{~h}$ post-infection (Figure 2A and Additional file 1: Table S2; $p<0.05)$. This finding suggests that the PspA mutant is impaired at replicating in the CNS in the early phase of infection. As previously reported [42], the rough strain FP23 was cleared from the brain by $24 \mathrm{~h}$ despite injection of animals with a high bacterial inoculum. As our PM model is characterised by the occurrence of both meningitis and sepsis [39],

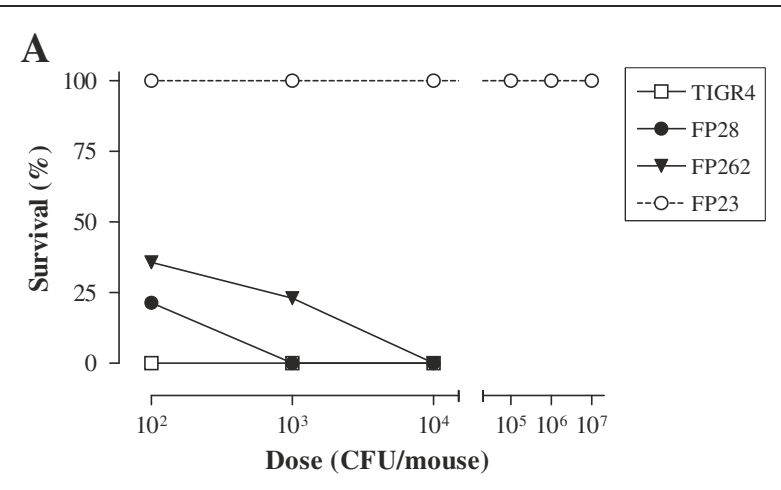

B

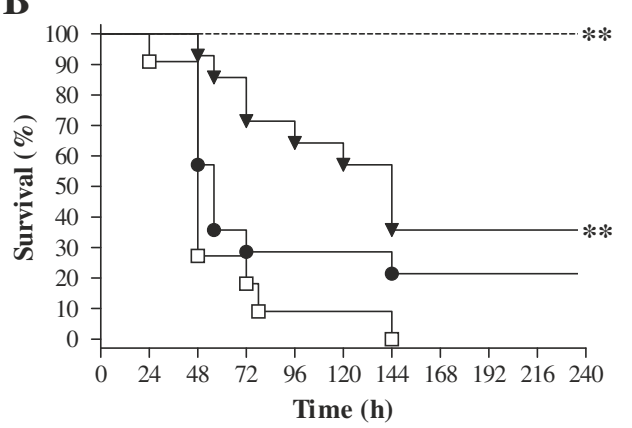

Figure 1 Survival curves of mice infected with S. pneumoniae strains. A. MF1 mice were infected by the i.c. route with $10^{2}, 10^{3}$ and $10^{4} \mathrm{cfu} /$ mouse of TIGR4 (wt, open squares), FP28 (PspC-, closed circles) and FP262 (PspA-, closed triangles). As a control, animals were also inoculated with different doses $\left(10^{2}-10^{7} \mathrm{cfu} / \mathrm{mouse}\right)$ of the unencapsulated mutant FP23 (open circles). Percent survival at different doses is shown. Data of two independent experiments are combined. B. Kaplan-Meyer curve of mouse survival following infection with $10^{2}$ cfu/mouse of TIGR4, FP28, FP262 and FP23. Mice were monitored for 10 days. Asterisks indicate statistical significance $\left({ }^{* *}, p<0.001\right.$; Log Rank test).

pneumococci were enumerated in the blood $24 \mathrm{~h}$ after i.c. infection with different bacterial doses $\left(10^{2}-10^{4} \mathrm{cfu} / \mathrm{mouse}\right)$. At the lowest inoculum of $10^{2} \mathrm{cfu} /$ mouse, the number of cfu counts was significantly different between mice infected with TIGR4 and those challenged with the mutants FP28, FP262 and FP23 with a progressively decreasing trend in mean $\log \mathrm{cfu} / \mathrm{ml}$ of blood (Figure $2 \mathrm{~B}$ and Additional file 1 : Table S3; $p<0.05$ ). In addition, significant differences could also be observed for the groups TIGR4-FP262 (at $10^{3} \mathrm{cfu} /$ mouse; $p<0.05$ ) and TIGR4-FP23 (at $10^{3}$ and $10^{4} \mathrm{cfu} /$ mouse, $p<0.05$ ), (Additional file 1: Table S3).

Evaluation of PM development in mice infected i.c. with S. pneumoniae strains

To assess whether the mutant strains were able to induce PM, mice were infected i.c. with $10^{4} \mathrm{cfu}$ of TIGR4 and the three mutants. Brains were collected $24 \mathrm{~h}$ after infection and subjected to histological analysis. Results 

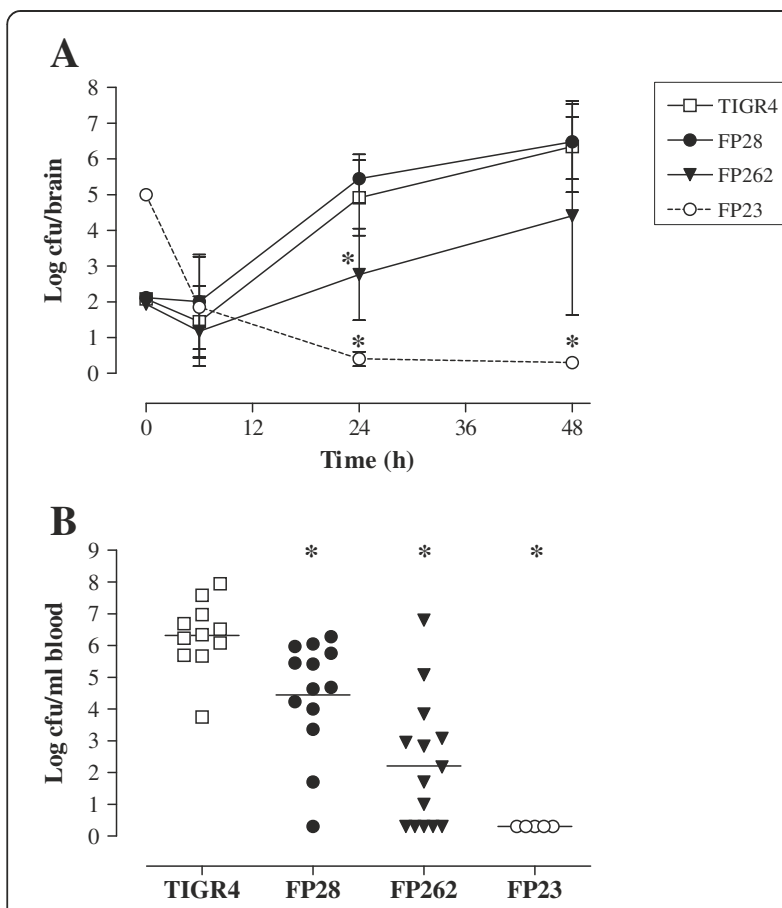

Figure 2 Bacterial loads in the brain and blood of mice i.c. challenged with S. pneumoniae. A. Mice were infected with $10^{2} \mathrm{cfu} /$ mouse of strains TIGR4 (wt, open squares), FP28 (PspC-, closed circles) and FP262 (PspA-, closed triangles). Control mice were inoculated with $10^{5} \mathrm{cfu} /$ mouse of the mutant FP23 (rough, open circles). Animals were sacrificed at different time-points $(6,24$ and $48 \mathrm{~h}$ ) after infection, and brains were collected to determine the viable counts. Results are represented as mean log $( \pm$ SD) cfu/brain over time. B. Animals were infected with $10^{2} \mathrm{cfu} /$ mouse of strains TIGR4, FP28, FP262 and FP23. Twenty-four h after infection, blood was collected and subjected to viable counts. Data are shown as log $\mathrm{cfu} / \mathrm{ml}$ of blood, and horizontal bars represent the mean cfu for each group. For both panels, asterisks indicate statistical significance $(*, p<0.05 ;$ Bootstrap method).

showed the presence of granulocytic infiltrations involving both the subarachnoid and ventricular spaces of the brain from mice inoculated with all the encapsulated strains, and no major differences could be observed between animals infected with TIGR4 and those challenged with the mutants FP28 and FP262 (data not shown). In contrast, no inflammation was detected in the brain of animals challenged with the rough FP23 mutant.

To further investigate this evidence, two groups of mice were inoculated with $10^{5} \mathrm{cfu}$ of TIGR4 and FP23 and euthanised 24 and $48 \mathrm{~h}$ later. Histological analysis of the brain from mice injected with TIGR4 showed severe inflammation characterised by massive infiltrations of PMN both on the meninges (Figure 3A) and in the ventricles, where accumulation of fibrin was also observed (Figure 3C). In contrast, the unencapsulated FP23 strain failed to cause inflammation on the meninges (Figure 3B) and in the ventricular spaces (Figure 3D). The TIGR4 strain also induced brain injury in the dentate gyrus of hippocampus, where shrunk neurons with picnotic nuclei were found (Figure 3E). No damage was found in samples from mice infected with FP23 (Figure 3F). A semi-quantitative analysis of brain inflammation and damage over time was carried out by determining the number of infiltrating PMN and the percentage of injured neurons in different brain regions, respectively, and by assigning each animal with an inflammation score (IS) and a damage score (DS). All mice infected with TIGR4 presented mild to severe inflammation that increased over time reaching a median IS (with IQR) of $12(2-12)$ at $48 \mathrm{~h}$ post-infection (Table 1). Likewise, all animals challenged with TIGR4 showed neuronal injury of different degrees peaking at $48 \mathrm{~h}$ with median DS (IQR) of 6 (1-8) (Table 1). In contrast, median IS and DS from mice infected with the FP23 strain were equal to 0 at both time-points (Table 1), although some mice still showed mild brain damage (data not shown). The above data were also confirmed by infecting animals with a larger dose of FP23 ( $10^{7} \mathrm{cfu} /$ mouse $)$ and analysing their brains at a later time-point $(72 \mathrm{~h}$ ) (data not shown). The brain tissue of control mice injected with PBS appeared normal. Differences in brain inflammation $(p<0.001)$ and neuronal damage $(p<0.05)$ between the groups infected with TIGR4 and FP23 were statistically significant.

\section{In vitro interaction of S. pneumoniae with microglia}

The susceptibility of TIGR4 and FP23 to BV2 microglial cells was recently investigated to elucidate the importance of the capsule in phagocytosis and killing by brain macrophages [42]. To understand the role of PspC and PspA in the interaction of S. pneumoniae with microglia, phagocytosis and intracellular survival of FP28 and FP262 were assessed in comparison with the parental strain TIGR4. The rough strain FP23 was employed as a control. By using a previously established fluorescent assay that allows to distinguish attached from internalised bacteria $[42,43]$, the number of phagocytic cells was measured at $3 \mathrm{~h}$ post-infection. Levels of phagocytosis were similar for all strains (Figure 4A and Additional file 1: Table S4), suggesting that in a serotype 4 background PspC and PspA do not significantly affect uptake by BV2 microglial cells. As the TIGR4 strain was shown to resist intracellular killing by BV2 cells despite being phagocytosed to the same extent of its isogenic unencapsulated strain FP23 [42], we investigated the behaviours of FP28 and FP262 in microglial cells. Bacteria associated with acidic phagosomes (phagolysosomes) were visualised by incubating Hoechst-labelled pneumococci with BV2 cells in the presence of LysoTracker, a marker of phagosome acidification. The number of phagolysosomes within each microglial cell was determined, and the percentage of colocalisation was calculated. At $3 \mathrm{~h}$ post-infection, the 

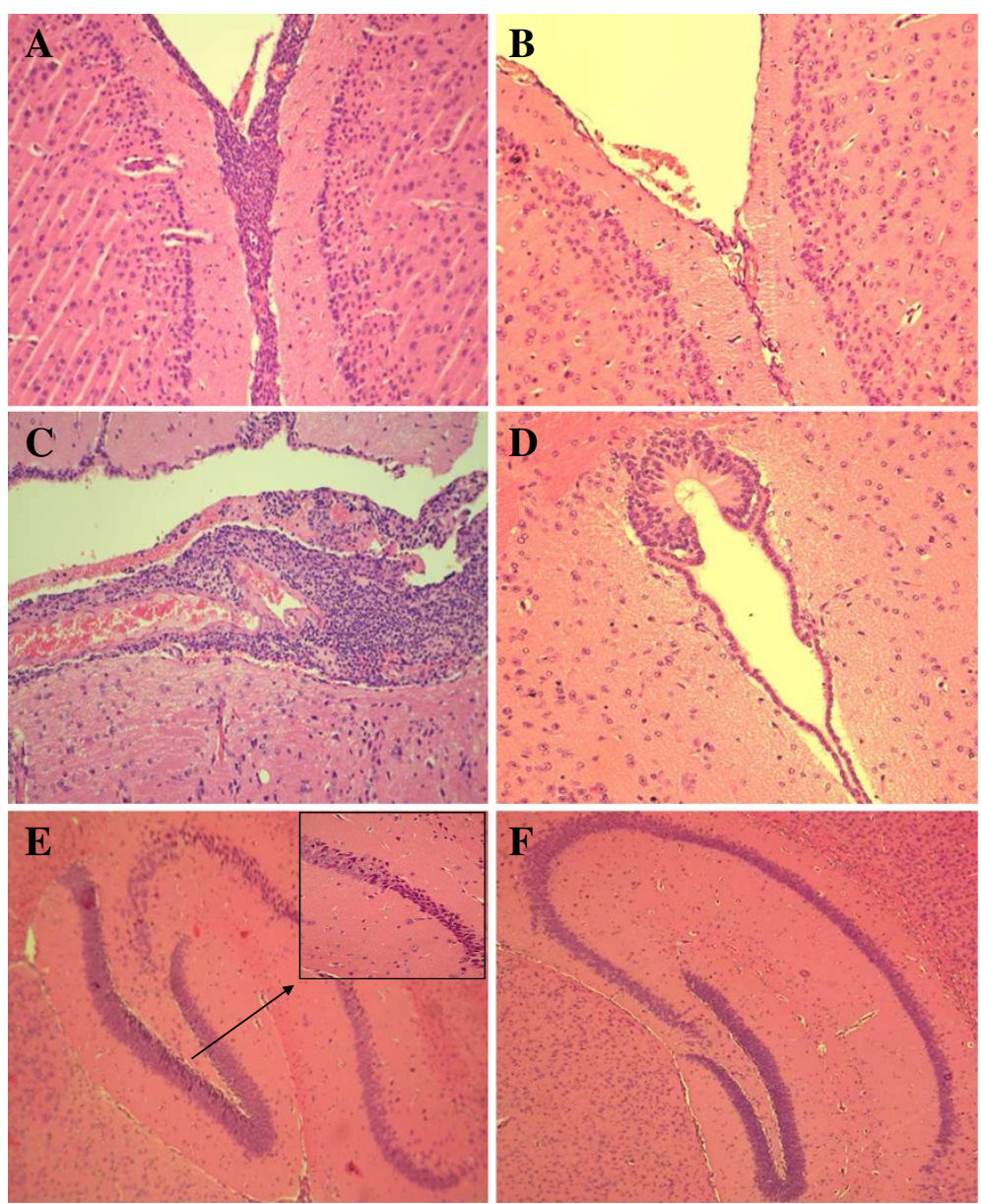

Figure 3 Brain histology of mice infected with TIGR4 or FP23. Mice were infected i.c. with $10^{5} \mathrm{cfu} / \mathrm{mouse}$ of TIGR4 or FP23 and sacrificed $24 \mathrm{~h}$ later. Brains were removed, fixed in formalin, embedded in paraffin, and stained with haematoxylin-eosin. The histopathological features of surface meninges (A, B), ventricular spaces (C, D) and hippocampus (E, F) of animals infected with TIGR4 (A, C, E) or FP23 (B, D, F) were

compared. A, C. Severe inflammation with massive PMN infiltrations over the inter-hemispheric fissure (A) and in the third ventricle (C). A bulk of fibrin is clearly visible in the ventricular space (C). B, D. No signs of inflammation on the meninges (B) and in a ventricle (D). E, F. Brain damage in the dentate gyrus (neuronal shrinkage is shown in the inset) of mice infected with TIGR4 (E), while the hippocampus of animals infected with FP23 was normal (F).

mean percentage of TIGR4-containing phagolysosomes was significantly lower $(p<0.05)$ than those of all mutant strains, suggesting that PspC and PspA may influence phagosome maturation in microglia (Figure $4 \mathrm{~B}$ and Additional file 1: Table S4). Finally, to examine the capability of FP28 and FP262 to survive within microglia, an intracellular survival assay was carried out by infecting BV2 cells with bacteria for $3 \mathrm{~h}$, followed by treatment with antibiotics, and then counting the number of intracellular surviving pneumococci at $4 \mathrm{~h}$ post-phagocytosis. Consistently with previous data [42], the survival index of FP23 was significantly lower (13folds; $p<0.05)$ than that of TIGR4. Survival of the PspA mutant FP262 was also significantly lower (4-folds; $p<0.05$ ) compared with the wt strain. No differences were observed between TIGR4 and FP28 (Figure 4C and Additional file 1: Table S4).

\section{Discussion}

Animal models of disease have significantly improved our knowledge on the interaction between $S$. pneumoniae and the host, and on the pathophysiological mechanisms involved in inflammation and brain damage during PM. Nevertheless, several issues remain to be clarified, including the role of different pneumococcal virulence factors in the disease. So far, only pneumolysin [29] and PavA [30] have been shown to contribute to PM development when tested via the i.c. route.

In this study, we used a mouse model of PM based on the inoculation of bacteria into the subarachnoid space [39] to test the pathogenicity of three different pneumococcal mutants in a type 4 background. PspA and PspC are well-known pneumococcal virulence factors $[1,2]$, but their impact on PM has not been investigated before. 


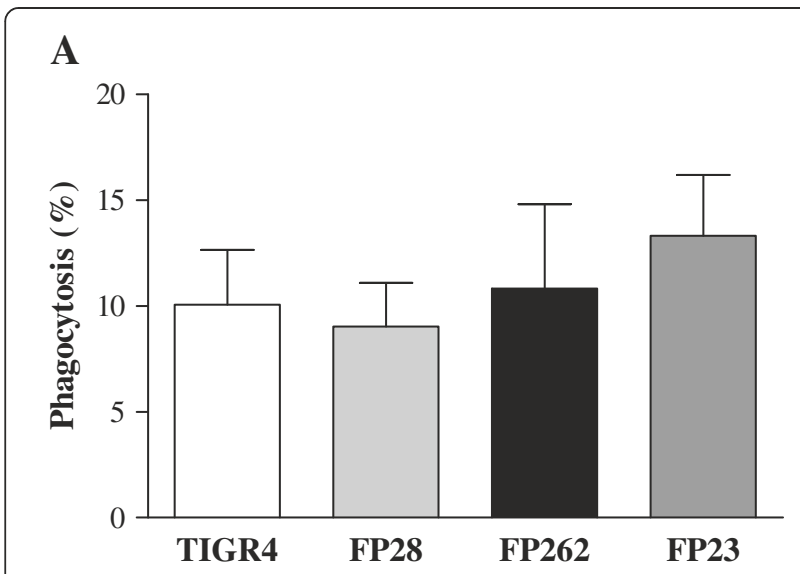

\section{B}

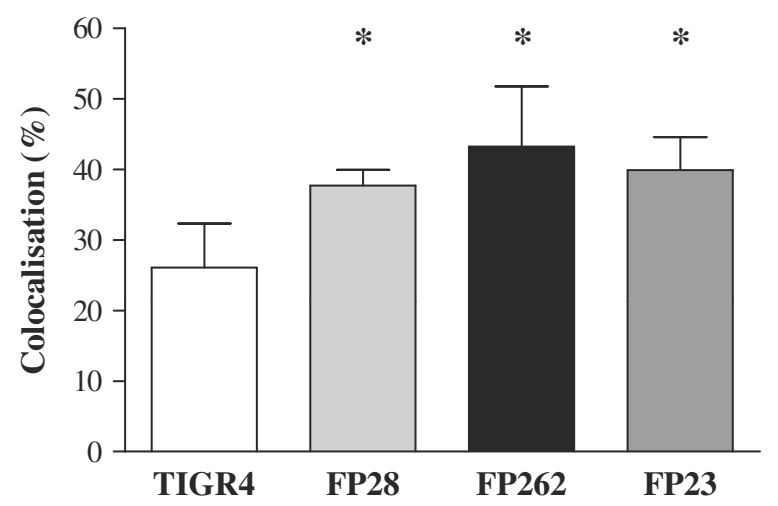

C

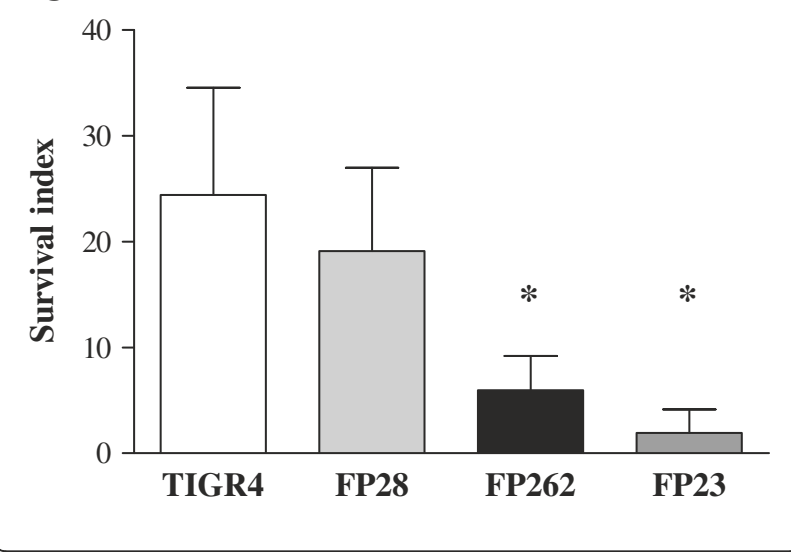

Figure 4 Phagocytosis and intracellular survival of $S$. pneumoniae strains in microglial cells. A. BV2 cells were infected for $3 \mathrm{~h}$ with bacteria (moi $=10$ ). A minimum of 200 BV2 cells were examined, and any cell containing one or more bacteria was considered as phagocytic. B. Quantification of acidic phagosomes in microglial cells infected with S. pneumoniae. Hoechst-labelled bacteria were exposed to BV2 cells $(\mathrm{moi}=10)$ for $3 \mathrm{~h}$, and the acidotropic dye LysoTracker Red DND-99 was added. Accumulation of the dye in phagosomes containing Hoechst-labelled bacteria was observed by epifluorescence microscopy. At least 200 BV2 cells were counted, and the number of cells with bacteria-containing acidic phagosomes was scored. The percentage of colocalisation was determined as the number of BV2 cells with pneumococcicontaining phagolysosomes over the total number of phagocytes. $\mathbf{C}$. Intracellular survival of pneumococcal strains within microglial cells. BV2 cells were infected for $3 \mathrm{~h}$ with S. pneumoniae strains ( $\mathrm{moi}=10$ ), washed to eliminate extracellular bacteria, and treated with antibiotics (time 0). After 4 h, BV2 were lysed, and viable counts were performed. The $\mathrm{SI}$ was calculated as the number of cfu detected at time $4 \mathrm{~h}$ divided by the cfu number at time $0 \mathrm{~h}$ postphagocytosis. For all above assays, data from $4-5$ independent experiments are shown as mean $\pm \mathrm{SD}$. Asterisks indicate statistical significance (*, $p<0.05$; Bootstrap method).

Mice infected with the PspA-deficient strain FP262 showed increased survival and prolonged time-to-death for all bacterial inocula as well as lower viable counts in both blood (at the doses of $10^{2}$ and $10^{3} \mathrm{cfu} /$ mouse) and brain (24 h post-infection) compared with TIGR4. Lower loads of FP262 in the blood may be due to decreased or delayed systemic spread of bacteria from the CNS and/ or higher clearance from the bloodstream. PspA plays a key role in immune escape of $S$. pneumoniae by interfering with complement deposition/activation [6-9]. Attenuation of PspA mutants in sepsis models is well documented [6,7,45-48], and the pspA gene was also shown to be upregulated in experimental sepsis [49]. Therefore, reduced virulence of FP262 in our PM model may be partially caused by its impaired virulence in the bloodstream. In addition, the ability of the FP262 strain to multiply in the CNS was initially hampered, as evidenced by the kinetics of viable bacterial counts in the brain. Nonetheless, the FP262 was still able to induce inflammation in the brain. In contrast, virulence of the PspC-deficient strain FP28 was moderately reduced compared with TIGR4, and the only condition able to unveil such difference was bacterial enumeration in the blood following infection with $10^{2} \mathrm{cfu} /$ mouse. Although PspC seems to have a limited impact in the PM model, our data do not exclude a possible role in crossing the $\mathrm{BBB}$, an early pathogenic step bypassed by i.c. infection. It was in fact suggested that $S$. pneumoniae tropism for the CNS may be partially due to PspC (CbpA), which participates in bacterial translocation from the blood to the CSF [50], most likely by binding to the laminin receptor present on the endothelial cells of BBB [51]. 
Once $S$. pneumoniae enters the CNS after crossing the $\mathrm{BBB}$ [52], the brain resident macrophages act as key effectors of initial innate immunity, by clearing bacteria and recruiting peripheral blood cells to the site of infection [33]. To investigate the role of PspA and PspC in the interaction with microglia, in vitro phagocytosis assays were performed using the well-established mouse microglial cell line BV2 [41]. Bacterial uptake by BV2 cells was comparable among the strains, in accordance with previous observations on TIGR4 and FP23 [42]. Nonetheless, when the fate of the different mutants inside microglia was analysed by evaluating colocalisation of intracellular pneumococci with acidic phagosomes and bacterial survival, both the unencapsulated strain FP23 and the PspA mutant FP262 showed significantly increased association with phagolysosomes and killing compared with the wt. As previously shown for the type 4 polysaccharide capsule [42], these data suggest that PspA may also participate in pneumococcal resistance to microglial killing, possibly by interfering with phagosome maturation. In contrast, survival of the PspC mutant FP28 in BV2 cells was similar to that of TIGR4, despite an increased association with phagolysosomes. This observation seems to disagree with a previous work reporting that the lack of $\mathrm{PspC}$ increased the susceptibility of pneumococcal killing by microglia [53]. Such discrepancy may be explained by the different pneumococcal strains employed in the assays, a serotype $3 \mathrm{PspC}$ mutant [53] and a serotype 4 PspC-deficient strain (this work), which are resistant and susceptible to phagocytosis by microglia, respectively. In summary, the data on PspA and PspC in our i.c. model (characterised by concurrent sepsis and meningitis) confirm previous reports on their key roles in experimental sepsis $[6,7,36,45-48,54]$. In addition, the decreased ability of FP262 of replicating in the brain at early time-points (24 h post-infection) together with its increased susceptibility to microglial killing also suggest that PspA may play a role in early stages of CNS infection by S. pneumoniae.

Rough strains are virtually unable to cause pneumococcal invasive disease [55], and to support this observation, fresh isolates from patients with pneumococcal infection are encapsulated [56]. However, early studies in a PM rabbit model showed that large inocula $\left(10^{7} \mathrm{cfu} /\right.$ $\mathrm{ml}$ ) of rough pneumococci could also be lethal, and that CSF inflammation could be induced by heat-killed unencapsulated S. pneumoniae (R6 strain) or their isolated cell walls, but not by heat-killed encapsulated pneumococci or their capsular polysaccharides $[23,24,57]$. Bacterial cell walls are potent inflammatory components, and the threshold of bioactivity of pneumococcal cell wall (PCW) corresponds to $\sim 10^{5} \mathrm{cfu} /$ $\mathrm{ml}$ of intact bacteria $[58,59]$. In our study, infection with up to $10^{7} \mathrm{cfu}$ (in $50 \mu \mathrm{l}$ ) of unencapsulated FP23 bacteria (corresponding to $2 \times 10^{8} \mathrm{cfu} / \mathrm{ml}$ of CSF) caused no meningeal inflammation or animal death ([42] and this work). Other than the PCW concentration, different factors may be responsible of the discrepancies observed, including the choice of different readouts to assess meningeal inflammation (i.e. cytochemical features of CSF versus histological analysis), the use of heat-inactivated versus live bacteria, and/or the type of $\mathrm{PCW}$ fragments released in the CSF of infected animals. As only specific motifs (i.e. trimeric stem peptides) of PCW are highly inflammatory [59], these structures may have not been accessible in FP23-infected mice, while being available in rabbits inoculated with heat-inactivated R6 bacteria [23]. Despite the lack of brain inflammation in mice injected with the FP23 mutant, a degree of neuronal damage was still found in the dentate gyrus of the hippocampus of some mice. As the effector mechanisms of neuronal damage in PM are both the host inflammatory response and the direct citotoxicity of bacterial components [19-21], the brain damage observed in mice infected with FP23 may be due to toxic molecules of $S$. pneumoniae, including pneumolysin and/or $\mathrm{H}_{2} \mathrm{O}_{2}$ in accordance to previous animal studies $[21,27,28]$.

\section{Conclusions}

The pathogenesis of PM is highly complex and multifactorial, and it is difficult to ascribe a precise role to a bacterial virulence factor in the disease. The results obtained with our i.c. model indicate a limited role for PspC, whereas PspA participates in PM pathogenesis possibly by interacting with microglial cells at an early phase of infection. The unencapsulated pneumococcal mutant failed to induce meningeal inflammation, brain injury and animal death even at high challenge doses, emphasising the pivotal role played by the capsule in invasive pneumococcal disease. The virulence factors evaluated in this study are either antigens of existing vaccines or strong candidates for vaccine development. The fact that they have been shown to contribute, to various degrees, to experimental PM support their use in current/future vaccine formulations against $S$. pneumoniae because vaccine efficacy may be enhanced by impairment of pneumococcal virulence. In conclusion, the data presented here may be relevant for translational research studies aimed at improving or developing effective and sustainable preventive measures against infectious diseases such as PM.

\section{Additional files}

Additional file 1: Detailed data with related statistical analysis on mouse survival (Table S1), viable counts in the brain over time (Table S2), viable counts in the blood $24 \mathrm{~h}$ post-infection (Table S3), and phagocytosis/colocalisation/survival assays using microglial cells (Table S4). 


\section{Abbreviations}

PspA: Pneumococcal surface protein A; PspC: Pneumococcal surface protein C; wt: Wild type; cfu: Colony forming units; PMN: Polymorphonuclear cells; CNS: Central nervous system; BBB: Blood-brain-barrier; CSF: Cerebrospinal fluid; PCW: Pneumococcal cell wall; IC: Intracranial; SI: Survival index IS: Inflammation score; DS: Damage score; FCS: Fetal calf serum; PBS: Phosphate buffered saline; PFA: Paraformaldehyde; IQR: Interquartile range; Cl: Confidence interval; SD: Standard deviation.

\section{Competing interests}

The authors declare that they have no competing interests.

\section{Authors' contributions}

$\mathrm{SR}$, co-ordination and design of the study, data analysis and interpretation, supervision of experimental work, writing of manuscript. AG, animal experiments and microbiological analysis. AP, statistical analyses. DC, animal experiments and manuscript revision. VB, animal experiments. SAT, histological analysis. BC, phagocytosis/survival experiments with microglia. $\mathrm{EB}$, co-ordination of phagocytosis experiments. $\mathrm{MRO}$, experimental design and critical reading of manuscript. SP, co-ordination of phagocytosis experiments, data evaluation, and manuscript revision. GP, co-ordination and design of the study, data evaluation. All authors read and approved the manuscript.

\section{Acknowledgements}

The work was supported by Italian MIUR 2008 (to G. Pozzi)

\section{Author details}

'Department of Medical Biotechnologies, Laboratory of Molecular Microbiology and Biotechnology (LA.M.M.B.), University of Siena and Siena University Hospital, Siena 53100, Italy. ${ }^{2}$ Department of Physiopathology, Experimental Medicine and Public Health, University of Siena, Siena, Italy. ${ }^{3}$ Department of Pathology, Siena University Hospital, Siena, Italy. ${ }^{4}$ Department of Diagnostics, Clinical and Public Health Medicine, University of Modena and Reggio Emilia (Unimore), Emilia-Romagna, Italy. ${ }^{5}$ Present address: Evans Medical Research Center, Boston University School of Medicine, 650 Albany Street, Boston, MA 02118, USA. ${ }^{6}$ Present address: Sanofi-Aventis S.p.a., Brindisi 72100, Italy.

Received: 27 May 2013 Accepted: 13 September 2013 Published: 24 September 2013

\section{References}

1. Kadioglu A, Weiser JN, Paton JC, Andrew PW: The role of Streptococcus pneumoniae virulence factors in host respiratory colonization and disease. Nat Rev Microbiol 2008, 6:288-301.

2. Mitchell AM, Mitchell TJ: Streptococcus pneumoniae: virulence factors and variation. Clin Microbiol Infect 2010, 16:411-418.

3. Brown EJ, Joiner KA, Cole RM, Berger M: Localization of complement component 3 on Streptococcus pneumoniae: anti-capsular antibody causes complement deposition on the pneumococcal capsule. Infect Immun 1983, 39:403-409.

4. Abeyta M, Hardy GG, Yother J: Genetic alteration of capsule type but not PspA type affects accessibility of surface-bound complement and surface antigens of Streptococcus pneumoniae. Infect Immun 2003, 71:218-225.

5. Hyams C, Camberlein E, Cohen JM, Bax K, Brown JS: The Streptococcus pneumoniae capsule inhibits complement activity and neutrophil phagocytosis by multiple mechanisms. Infect Immun 2010, 78:704-715.

6. Tu AH, Fulgham RL, McCrory MA, Briles DE, Szalai AJ: Pneumococcal surface protein A inhibits complement activation by Streptococcus pneumoniae. Infect Immun 1999, 67:4720-4724.

7. Ren B, McCrory MA, Pass C, Bullard DC, Ballantyne CM, Xu Y, Briles DE, Szalai AJ: The virulence function of Streptococcus pneumoniae surface protein $A$ involves inhibition of complement activation and impairment of complement receptor-mediated protection. J Immunol 2004, 173:7506-7512.

8. Ren B, Szalai A, Hollingshead SK, Briles DE: Effects of PspA and antibodies to PspA on activation and deposition of complement on the pneumococcal surface. Infect Immun 2004, 72:114-122.

9. Mukerji R, Mirza S, Roche AM, Widener RW, Croney CM, Rhee DK, Weiser JN, Szalai AJ, Briles DE: Pneumococcal surface protein A inhibits complement deposition on the pneumococcal surface by competing with the binding of C-reactive protein to cell-surface phosphocholine. J Immunol 2012, 189:5327-5335

10. Hammerschmidt S, Bethe G, Remane PH, Chhatwal GS: Identification of pneumococcal surface protein $A$ as a lactoferrin-binding protein of Streptococcus pneumoniae. Infect Immun 1999, 67:1683-1687.

11. Dave S, Carmicle S, Hammerschmidt S, Pangburn MK, Mc Daniel LS: Dual roles of PspC, a surface protein of Streptococcus pneumoniae, in binding human secretory IgA and factor H. J Immunol 2004, 173:471-477.

12. Hammerschmidt S, Tilling MP, Wolff S, Vaerman JP, Chhatwal GS: Speciesspecific binding of human secretory component to SpsA protein of Streptococcus pneumonie via a hexapeptide motif. Mol Microbiol 2000, 36:726-736.

13. Zhang JR, Mostov KE, Nanno M, Shimida S, Ohwaki M, Tuomanen E: The polymeric immunoglobulin receptor translocates pneumococci across human nasopharyngeal epithelial cells. Cell 2000, 102:827-837.

14. Elm C, Braathen R, Bergmann S, Frank R, Vaerman JP, Kaetzel CS, Chhatwal GS, Johansen FE, Hammerschmidt S: Ectodomains 3 and 4 of human polymeric Immunoglobulin receptor (hplgR) mediate invasion of Streptococcus pneumoniae into the epithelium. J Biol Chem 2004, 279:6296-6304.

15. Smith BL, Hostetter MK: C3 as substrate for adhesion of Streptococcus pneumoniae. J Infect Dis 2000, 182:497-508.

16. Janulczyk R, lannelli F, Sjöholm AG, Pozzi G, Björck L: Hic, a novel surface protein of Streptococcus pneumoniae that interferes with complement function. J Biol Chem 2000, 275:37257-37263.

17. Jarva H, Janulczyk R, Hellwage J, Zipfel PF, Björck L, Meri S: Streptococcus pneumoniae evades complement attack and opsonophagocytosis by expressing the $p s p C$ locus-encoded Hic protein that binds to short consensus repeats 8-11 of factor H. J Immunol 2002, 168:1886-1894.

18. Duthy TG, Ormsby RJ, Giannakis E, Ogunniyi DA, Stroeher UH, Paton JC, Gordon DL: The human complement regulator factor $\mathrm{H}$ binds pneumococcal surface protein PspC via short consensus repeats 13 to 15 . Infect Immun 2002, 70:5604-5611.

19. Mook-Kanamori BB, Geldhoff M, van der Poll T, van de Beek D: Pathogenesis and pathophysiology of pneumococcal meningitis. Clin Microbiol Rev 2011, 24:557-591.

20. Koedel U, Klein M, Pfister HW: New understandings on the pathophysiology of bacterial meningitis. Curr Opin Infect Dis 2010, 23:217-223.

21. Gerber J, Nau R: Mechanisms of injury in bacterial meningitis. Curr Opin Neurol 2010, 23:312-318.

22. Klein M, Obermaier B, Angele B, Pfister HW, Wagner H, Koedel U, Kirschning $\mathrm{CJ}$ : Innate immunity to pneumococcal infection of the central nervous system depends on toll-like receptor (TLR) 2 and TLR4. J Infect Dis 2008, 198:1028-1036.

23. Tuomanen $\mathrm{E}$, Tomasz A, Hengstler B, Zak O: The relative role of bacterial cell wall and capsule in the induction of inflammation in pneumococcal meningitis. J Infect Dis 1985, 151:535-540.

24. Tuomanen E, Liu H, Hengstler B, Zak O, Tomasz A: The induction of meningeal inflammation by components of the pneumococcal cell wall. $J$ Infect Dis 1985, 151:859-868.

25. Hirst RA, Sikand KS, Rutman A, Mitchell TJ, Andrew PW, O'Callaghan C: Relative roles of pneumolysin and hydrogen peroxide from Streptococcus pneumoniae in inhibition of ependymal ciliary beat frequency. Infect Immun 2000, 68:1557-1562.

26. Zysk G, Schneider-Wald BK, Hwang JH, Bejo L, Kim KS, Mitchell TJ, Hakenbeck R, Heinz HP: Pneumolysin is the main inducer of cytotoxicity to brain microvascular endothelial cells caused by Streptococcus pneumoniae. Infect Immun 2001, 69:845-852.

27. Braun JS, Sublett JE, Freyer D, Mitchell TJ, Cleveland JL, Tuomanen El, Weber JR: Pneumococcal pneumolysin and $\mathrm{H}_{2} \mathrm{O}_{2}$ mediate brain cell apoptosis during meningitis. J Clin Invest 2002, 109:19-27.

28. Winter AJ, Comis SD, Osborne MP, Tarlow MJ, Stephen J, Andrew PW, Hill J, Mitchell TJ: A role for pneumolysin but not neuraminidase in the hearing loss and cochlear damage induced by experimental pneumococcal meningitis in guinea pigs. Infect Immun 1997, 65:4411-4418.

29. Wellmer A, Zysk G, Gerber J, Kunst T, von Mering M, Bunkowski S, Eiffert H, Nau R: Decreased virulence of a pneumolysin-deficient strain of Streptococcus pneumoniae in murine meningitis. Infect Immun 2002, 70:6504-6508 
30. Pracht D, Elm C, Gerber G, Bergmann S, Rohde M, Seiler M, Kim KS, Jenkinson HF, Nau R, Hammerschmidt S: PavA of Streptococcus pneumoniae modulates adherence, invasion, and meningeal inflammation. Infect Immun 2005, 73:2680-2689.

31. Vilhardt F: Microglia: phagocyte and glia cell. Int J Biochem Cell Biol 2005, 37:17-21

32. Hauwel M, Furon E, Canova C, Griffiths M, Neal J, Gasque P: Innate (inherent) control of brain infection, brain inflammation and brain repair: the role of microglia, astrocytes, "protective" glial stem cells and stromal ependymal cells. Brain Res Rev 2005, 48:220-233.

33. Rock RB, Gekker G, Hu S, Sheng WS, Cheeran M, Lokensgard JR, Peterson PK: Role of microglia in central nervous system infections. Clin Microbiol Rev 2004, 17:942-964.

34. Horton RM, Cai Z, Ho SN, Pease LR: Gene splicing by overlap extension: tailor-made genes using the polymerase chain reaction. Biotechniques 1990, 8:528-535

35. Ali F, Lee ME, lannelli F, Pozzi G, Mitchell TJ, Read RC, Dockrell DH: Streptococcus pneumoniae-associated human macrophage apoptosis after bacterial internalization via complement and Fcgamma receptors correlates with intracellular bacterial load. J Infect Dis 2003, 188:1119-1131.

36. lannelli F, Chiavolini D, Ricci S, Oggioni MR, Pozzi G: Pneumococcal surface protein C contributes to sepsis caused by Streptococcus pneumoniae in mice. Infect Immun 2004, 72:3077-3080.

37. Pearce BJ, lannelli F, Pozzi G: Construction of new unencapsulated (rough) strains of Streptococcus pneumoniae. Res Microbiol 2002, 153:243-247.

38. Oggioni MR, Memmi G, Maggi T, Chiavolini D, lannelli F, Pozzi G: Pneumococcal zinc metalloproteinase $\mathrm{ZmpC}$ cleaves human matrix metalloproteinase 9 and is a virulence factor in experimental pneumonia. Mol Microbiol 2003, 49:795-805.

39. Chiavolini D, Tripodi S, Parigi R, Oggioni MR, Blasi E, Cintorino M, Pozzi G, Ricci S: Method for inducing experimental pneumococcal meningitis in outbred mice. BMC Microbiol 2004, 4:36.

40. Sandgren A, Albiger B, Orihuela CJ, Tuomanen E, Normark S, HenriquesNormark B: Virulence in mice of pneumococcal clonal types with known invasive disease potential in humans. J Infect Dis 2005, 192:791-800.

41. Blasi $E$, Barluzzi $R$, Bocchini $V$, Mazzolla R, Bistoni F: Immortalization of murine microglial cells by a v-raf/v-myc carrying retrovirus. J Neuroimmuno/ 1990, 27:229-237.

42. Peppoloni S, Ricci S, Orsi CF, Colombari B, De Santi MM, Messinò M, Fabio G, Zanardi A, Righi E, Braione V, et al: The encapsulated strain TIGR4 of Streptococcus pneumoniae is phagocytosed but is resistant to intracellular killing by mouse microglia. Microbes Infect 2010, 12:990-1001.

43. Peppoloni S, Colombari B, Beninati C, Felici F, Teti G, Speziale P, Ricci S, Ardizzoni A, Manca L, Blasi E: The Spr1875 protein confers resistance to the microglia-mediated killing of Streptococcus pneumoniae. Microb Pathog 2013, 59-60:42-47.

44. Efron B, Tibshirani RJ: An introduction to the Bootstrap. New York: Chapman and Hall; 1994

45. McDaniel LS, Yother J, Vijayakumar MN, McGarry L, Guild WR, Briles DE: Use of insertional inactivation to facilitate studies of biological properties of pneumococcal surface protein A (PspA). J Exp Med 1987, 165:381-394.

46. Berry AM, Paton JC: Additive attenuation of virulence of Streptococcus pneumoniae by mutation of the genes encoding pneumolysin and other putative pneumococcal virulence proteins. Infect Immun 2000, 68:133-140

47. Yuste J, Botto M, Paton JC, Holden DW, Brown JS: Additive inhibition of complement deposition by pneumolysin and PspA facilitates Streptococcus pneumoniae septicemia. J Immunol 2005, 175:1813-1819.

48. Quin LR, Moore QC III, McDaniel LS: Pneumolysin, PspA, and PspC contribute to pneumococcal evasion of early innate immune responses during bacteremia in mice. Infect Immun 2007, 75:2067-2070.

49. Ogunniyi DA, Giammarinaro P, Paton JC: The genes encoding virulenceassociated proteins and the capsule of Streptococcus pneumoniae are upregulated and differentially expressed in vivo. Microbiology 2002, 148:2045-2053.

50. Orihuela CJ, Gao G, Francis KP, Yu J, Tuomanen El: Tissue-specific contributions of pneumococcal virulence factors to pathogenesis. $J$ Infect Dis 2004, 190:1661-1669.
51. Orihuela CJ, Mahdavi J, Thornton J, Mann B, Wooldridge KG, Abouseada N, Oldfield NJ, Self T, Ala'Aldeen DA, Tuomanen El: Laminin receptor initiates bacterial contact with the blood brain barrier in experimental meningitis models. J Clin Invest 2009, 119:1638-1646.

52. Ring A, Weiser JN, Tuomanen El: Pneumococcal trafficking across the blood-brain barrier. Molecular analysis of a novel bidirectional pathway. J Clin Invest 1998, 102:347-360.

53. Peppoloni S, Colombari B, Neglia R, Quaglino D, lannelli F, Oggioni MR, Pozzi G, Blasi E: The lack of pneumococcal surface protein C (PspC) increases the susceptibility of Streptococcus pneumoniae to the killing by microglia. Med Microbiol Immunol 2006, 195:21-28.

54. Kerr AR, Paterson GK, McCluskey J, lannelli F, Oggioni MR, Pozzi G, Mitchell $\mathrm{TJ}$ : The contribution of PspC to pneumococcal virulence varies between strains and is accomplished by both complement evasion and complement-independent mechanisms. Infect Immun 2006, 74:5319-5324.

55. Griffith F: The significance of pneumococcal types. J Hyg 1928, 27:113-159.

56. Austrian R: Some observations on the pneumococcus and on the current status of pneumococcal disease and its prevention. Rev Infect Dis 1981, 3:S1-S17.

57. Tomasz A, Saukkonen K: The nature of cell wall-derived inflammatory components of pneumococci. Pediatr Infect Dis 1989, 8:902-903.

58. Weber JR, Moreillon P, Tuomanen El: Innate sensors for Gram-positive bacteria. Curr Opin Immunol 2003, 15:408-415.

59. Moreillon P, Majcherczyk PA: Proinflammatory activity of cell-wall constituents from Gram-positive bacteria. Scand J Infect Dis 2003, 35:632-641.

doi:10.1186/1471-2334-13-444

Cite this article as: Ricci et al:: Contribution of different pneumococcal virulence factors to experimental meningitis in mice. BMC Infectious Diseases 2013 13:444.

\section{Submit your next manuscript to BioMed Central and take full advantage of:}

- Convenient online submission

- Thorough peer review

- No space constraints or color figure charges

- Immediate publication on acceptance

- Inclusion in PubMed, CAS, Scopus and Google Scholar

- Research which is freely available for redistribution

Submit your manuscript at www.biomedcentral.com/submit
C) BioMed Central 